case report

\title{
Angiosarcoma of the liver after multimodality therapy for gallbladder carcinoma
}

\author{
Maikel Botros, J. Fernando Quevedo, Robert C. Miller \\ Department of Radiation Oncology and the Department of Oncology, \\ Mayo Clinic, Rochester, Minnesota, USA
}

\begin{abstract}
Background. Treatment-induced angiosarcoma is a rare complication of cancer therapy and is best known for arising on the chest wall after a mastectomy. We report an unusual case of a treatment-induced angiosarcoma developing in the liver after adjuvant radiotherapy for gallbladder carcinoma.

Case report. Radiation-induced angiosarcoma of the liver developed in a 46-year-old woman after radiotherapy for stage IIB (T3N1M0) adenocarcinoma of the gallbladder in 1991. The patient subsequently underwent resection, postoperative external beam radiotherapy, and multiagent chemotherapy. Several severe late adverse effects developed, including duodenal obstruction with fistula formation and chronic mesenteric ischemia secondary to occlusion of the superior mesenteric artery. Six years after her gallbladder resection and adjuvant treatment, a fatal grade 4 of 4 angiosarcoma of the liver developed within the radiation field. Conclusions. To our knowledge, this is the first case of radiation-induced angiosarcoma of the liver after radiotherapy for gallbladder carcinoma. Normal organ dose-volume limits should be considered carefully when delivering a course of external beam radiotherapy in the upper abdomen.
\end{abstract}

Key words: angiosarcoma; gallbladder carcinoma; liver; radiotherapy toxicity

\section{Introduction}

Angiosarcomas are rare malignant tumors arising from the vasculature. ${ }^{1}$ Angiosarcomas of the liver occur even less commonly; about 25 cases are report-

Received 26 January 2009

Accepted 14 February 2009

Correspondence to: Robert C. Miller, MD, MS, Department of Radiation Oncology, Mayo Clinic, 200 First Street SW, Rochester, MN 55905. E-mail: miller. robert@mayo.edu

Conflict of interest: The authors have no conflicts of interest to disclose. ed in the United States every year. Most angiosarcomas arising from the liver are secondary to long-term exposure to thorium dioxide, arsenic, or vinyl chloride. ${ }^{2}$ To our knowledge, radiotherapy-induced liver angiosarcomas have not been previously reported. The purpose of this paper is to describe the first case of a radiotherapyinduced liver angiosarcoma.

\section{Case report}

A 46-year-old woman presented with acute pain in the right upper quadrant of her 
abdomen in 1991. Ultrasonography at that time revealed a thickened gallbladder wall. A computed tomographic (CT) scan of the abdomen demonstrated a mass arising in the gallbladder and extending beyond the gallbladder wall into the porta hepatis and the left hepatic lobe. Her staging work-up, which included a barium enema study, sigmoidoscopy, and pelvic ultrasonography, revealed no sites of metastatic disease. Levels of $\alpha$-fetoprotein and cancer antigen 125 were within normal limits. Carcinoembryonic antigen (CEA) and alkaline phosphatase values were slightly elevated at $4.4 \mathrm{ng} / \mathrm{mL}$ and 126 units/L, respectively. The patient had a 20-pack-year history of smoking. There was no familial history of cancer or other genetic conditions.

The patient underwent a cholecystectomy and partial hepatectomy at another institution. Pathologic review of the resected specimen revealed a well- to moderately differentiated mucinous adenocarcinoma of the gallbladder, extending beyond the gallbladder wall to involve the liver capsule, stage IIB (T3N1M0). Three of 3 evaluated lymph nodes were involved with tumor. The patient's postoperative recovery was unremarkable and adjuvant therapy was recommended, given the primary tumor's adherence to the liver and the involved lymph nodes. The patient elected to receive adjuvant therapy. A course of external beam radiotherapy to a total dose of $54 \mathrm{~Gy}$ in 1.8-Gy daily fractions was administered using a 4-field technique. The patient received adjuvant chemotherapy consisting of fluorouracil, leucovorin, and mitomycin $\mathrm{C}$ concurrently with her radiotherapy. Her radiotherapy course was marked by grade 3 gastrointestinal tract toxicity, and she required hospital admission and support with intravenous hydration secondary to nausea, vomiting, and dehydration.

Over the next year, the patient had progressive upper abdominal pain with con- comitant weight loss. Approximately 1 year after radiotherapy, an esophagogastroduodenoscopy revealed friable gastritis involving the distal antrum along with duodenitis involving the duodenal bulb, secondary to the radiation effect. In the next year, the patient continued to experience symptoms of persistent gastritis and duodenitis, and the following year she underwent a vagotomy and gastrojejunostomy to bypass a stricture of the duodenum, with an associated fistula. Four years after radiotherapy, in 1995, a diagnosis of chronic mesenteric ischemia was made. Mesenteric angiography revealed a $3-\mathrm{cm}$ proximal occlusion of the superior mesenteric artery. She underwent aortic-to-superior mesenteric artery bypass at that time, and her symptoms resolved.

Six years after radiotherapy, in 1997, right upper quadrant pain and persistent nocturnal fevers up to $38.9^{\circ} \mathrm{C}$ developed. On clinical examination, she had right upper quadrant tenderness with abdominal distension and a palpable mass in the right upper quadrant. A CT scan subsequently revealed a $12-\mathrm{cm}$ mass involving the anterior segment of the right hepatic lobe and medial left segment of the left hepatic lobe, with additional satellite lesions within the left lateral hepatic segment. The tumor extended into the porta hepatis, with occlusion and thrombus of the anterior segmental division of the right portal vein, and involved the prior gallbladder fossa. There was no evidence of neoplastic spread outside the liver. A biopsy revealed a spindle cell neoplasm consistent with angiosarcoma, grade 4 of 4 . Immunohistochemical stains for CD31 and vimentin were positive and negative for keratin, CEA, and S-100.

The patient was referred to Mayo Clinic in Rochester, Minnesota, for evaluation and treatment at this point. She was not considered a surgical candidate because of the multifocal presence of tumor within the liver. A symptomatic thrombus of her supe- 
rior mesenteric artery bypass graft developed approximately 14 days after the diagnosis of angiosarcoma. A course of salvage chemotherapy, consisting of ifosfamide, mitomycin $\mathrm{C}$, doxorubicin, and cisplatin, was recommended, but the patient succumbed to fatal mesenteric ischemia before initiation of any systemic therapy.

\section{Discussion}

Gallbladder carcinoma is a rare malignancy. The prognosis is often poor because of the advanced stage at the time of diagnosis. Surgery remains the treatment of choice for gallbladder carcinoma. ${ }^{3}$ For gallbladder carcinoma that is limited to the gallbladder mucosa, cholecystectomy is often sufficient to eradicate all malignant disease. However, for locally and regionally advanced disease, a radical cholecystectomy is recommended. For patients in whom an incidental carcinoma is found at the time of routine cholecystectomy, re-resection with abdominal exploration and lymph node dissection is often considered.

Given the high risk for systemic as well as regional recurrence in locally advanced tumors, we consider adjuvant therapy after resection of tumors that are locally extensive, invade adjacent organs, or involve regional lymph nodes. No randomized trials have been performed to quantify the benefit of such adjuvant therapy, however. ${ }^{4}$ Retrospective studies have suggested that postoperative chemoradiotherapy has a survival benefit in patients with high-risk tumors, ie, tumors that show extensive local infiltration outside the wall of the gallbladder or those with regional lymph node involvement, in the absence of metastatic disease. ${ }^{4-6}$ A study from Mayo Clinic, published in 2002, described 12 patients who underwent adjuvant chemoradiotherapy for gallbladder cancer after resection with negative margins and had 5-year survival of $64 \% .^{5}$ Historical surgical controls from Mayo Clinic and other institutions had 5 -year survival of $33 \%$ for patients treated with surgery alone. ${ }^{5}$ A more recent evaluation at Mayo Clinic showed survival rates were equivalent for patients undergoing surgery and for those undergoing surgery and adjuvant radiotherapy. ${ }^{4}$ However, the majority of patients who underwent resection alone did so for stage I gallbladder carcinoma $(88 \%)$, whereas patients receiving adjuvant therapy had more advanced stages. Despite having more advanced stages, patients receiving adjuvant therapy, primarily concurrent fluorouracil and external beam radiotherapy, had a survival rate comparable to those undergoing surgery alone for stage I disease. ${ }^{4} \mathrm{~A}$ recent large Surveillance, Epidemiology, and End Results analysis showed a benefit in overall survival rates for selected patients undergoing postoperative adjuvant chemotherapy. ${ }^{7}$ Given the relative rarity of the disease in the Western world, however, it is unlikely that a definitive randomized trial will address this question. Retrospective studies from Mayo Clinic and elsewhere suggest the benefit of this treatment, but these studies are limited by known biases regarding patient selection with respect to performance status and other factors.

The difficulties encountered in case studies highlight the potential pitfalls in delivering external beam radiotherapy in the right upper quadrant. Modern image-guided radiotherapy techniques, coupled with 4-dimensional CT-based treatment planning, now provide radiation oncologists with tools to avoid excess doses in critical organs. The chronic adverse effects in the patient described here-duodenal strictures, chronic gastritis, and arterial ischemia-are known complications resulting from highdose and large-volume irradiation of the organs in the gastrointestinal tract. Also, 
the risk of chronic, radiation-induced liver disease must be considered in treatment planning. ${ }^{8}$

The long-term adverse effects of radiation on the stomach may include gastritis with stenosis, dyspepsia, and ulceration. Gastritis may appear from 1 to 12 months after radiation treatment and is usually accompanied by antral stenosis. Endoscopy may also reveal atrophy of the mucosal wall. The onset of dyspepsia varies and may occur up to 4 years after treatment. Radiation-induced gastric ulcerations may heal spontaneously but are usually accompanied by fibrosis. Management of chronic gastritis has not been extensively studied. Some physicians elect to prescribe antiulcer medications such as $\mathrm{H}_{2}$-receptor blockers. $^{9}$

The volume of small bowel receiving high doses of radiotherapy is typically the limiting factor in radiotherapy planning for the upper abdomen. Radiation effects include fibrosis and chronic ischemia after vascular injury. Multifocal adhesions and areas of stenosis can occur in a dose-dependent fashion. ${ }^{9}$ Radiotherapy can decrease the number of mucosal stem cells in the small intestine, causing inflammation and shortening of the villi and leading to a decrease in intestinal absorption. Additionally, radiation can induce, independent of injury to the villi, an inflammatory cytokine cascade in which proinflammatory cytokines such as tumor necrosis factor $\alpha$ and interleukins 1 and 6 contribute to a cycle of initiation of injury, upregulation with generation of messenger agents, and ulceration with further inflammation. ${ }^{10}$ Vascular damage caused by radiation may involve hyalinized thickening and foam cell deposition in small-sized $(<100 \mu \mathrm{m})$ and medium-sized (100-500 $\mu \mathrm{m})$ arteries. Lymphocytic infiltration can occur in the tunica media and tunica adventitia. These changes are typical of small-vessel injury from radiotherapy and may, in rare cases, occur in larger vessels. ${ }^{11}$ Symptoms of enteritis may take years to develop after irradiation of the abdomen, but the reported median is 8 to 12 months. ${ }^{9}$ Toxicity is strongly correlated with the volume of small bowel irradiated in conjunction with the dose. 9,12,13 $^{-1,}$

At Mayo Clinic, our current approach to postoperative treatment of locally advanced gallbladder carcinomas involves careful reevaluation of the patient's nutritional status and cancer staging status approximately 1 month after surgery, followed by consideration of concurrent radiotherapy and chemotherapy. For patients who have adequately recovered from their resection and who have no evidence of metastatic disease, either at surgery or at the time of restaging after surgery, consideration is given to systemic chemotherapy with gemcitabine as initial treatment. This is followed by concurrent continuous infusional fluorouracil chemotherapy with $50.4 \mathrm{~Gy}$ in 1.8-Gy daily fractions of conformal external beam radiotherapy to the gallbladder fossa and regional lymphatics. Then gemcitabinebased chemotherapy is resumed 1 month after completion of radiotherapy. This regimen is similar to the adjuvant therapy delivered postoperatively with gemcitabine for pancreatic cancer during the Radiation Therapy Oncology Group 97-04 trial ${ }^{14}$, with the exception of a change in radiotherapy target volume. A total radiation dose of 50.4 Gy is delivered to the tumor bed and structures involved by the tumor at the time of resection, with a lower dose of $45.0 \mathrm{~Gy}$ delivered to uninvolved lymph node regions at risk in the porta hepatis, pancreatoduodenal lymph nodes, and celiac axis. The radiotherapy must be carefully planned to provide adequate planning target volume (PTV) coverage $(95 \%$ of the PTV receiving $\geq 50.4 \mathrm{~Gy}$ ) while respecting liver tolerance (mean liver dose in patients without preexisting liver disease $\leq 34 \mathrm{~Gy}$; volume of liver 
receiving $\geq 30$ Gy is $\leq 50 \%$ ); kidney tolerance (volume of kidney receiving $\geq 18 \mathrm{~Gy}$ is $\leq 33 \%$ for at least 1 kidney and preferably both); and limiting maximum small bowel dose to no more than 52 Gy. Radiotherapy beam arrangements typically consist of opposed laterals, 2 anterior oblique angles $45^{\circ}$ off vertical, a noncoplanar anterior beam angled $30^{\circ}$ superior, and a noncoplanar posterior beam angled $20^{\circ}$ toward the feet, with the angle of the posterior beam limited by the linear accelerator and couch design. A 4-dimensional CT scan for radiotherapy planning is performed at the time of simulation to track the target volume at risk with the breathing cycle.

Ionizing radiation can produce sarcomas in irradiated tissues. Cahan et al. ${ }^{15}$ have published a well-recognized set of criteria to describe radiotherapy-induced sarcomas. First, the sarcoma should arise in an area that was previously irradiated. Second, a period of latency must exist between the radiotherapy and onset of the sarcoma. Finally, histologic confirmation of the sarcoma should be obtained. ${ }^{16}$ Applying the criteria of Cahan et al. ${ }^{15}$, the angiosarcoma reported here would meet the criteria for a radiation-induced malignancy. Previously, angiosarcomas have been reported in organs such as the heart, liver, spleen, and adrenal glands and have had a poor prognosis. Angiosarcomas have also been reported after mastectomy in patients with breast cancer due to long-standing lymphedema (Stewart-Treves syndrome). ${ }^{1}$

In summary, the role of adjuvant therapy in the treatment of locally advanced, resected gallbladder carcinoma remains to be optimally defined. On the basis of limited retrospective evidence, chemotherapy, with or without concurrent chemoradiotherapy, may reduce the risk of recurrence for patients with risk factors for local and regional recurrence. The chronic adverse effects of treatment emphasize the need for careful consideration of normal-organ dose-volume limits when delivering a course of external beam radiotherapy in the upper abdomen.

\section{References}

1. Koch M, Nielsen GP, Yoon SS. Malignant tumors of blood vessels: angiosarcomas, hemangioendotheliomas, and hemangioperictyomas. J Surg Oncol 2008; 97: 321-9.

2. Centers for Disease Control and Prevention (CDC). Epidemiologic notes and reports: angiosarcoma of the liver among polyvinyl chloride workers-Kentucky. 1974. MMWR Morb Mortal Wkly Rep. 1997; 46: 97-101.

3. Reid KM, Ramos-De la Medina A, Donohue JH. Diagnosis and surgical management of gallbladder cancer: a review. J Gastrointest Surg 2007; 11: 671-81.

4. Gold DG, Miller RC, Haddock MG, Gunderson LL, Quevedo JF, Donohue JH, et al. Adjuvant therapy for gallbladder carcinoma: the Mayo Clinic experience. Int J Radiat Oncol Biol Phys 2009 Mar 16. [Epub ahead of print]

5. Kresl JJ, Schild SE, Henning GT, Gunderson LL, Donohue J, Pitot $\mathrm{H}$, et al. Adjuvant external beam radiation therapy with concurrent chemotherapy in the management of gallbladder carcinoma. Int J Radiat Oncol Biol Phys 2002; 52: 167-75.

6. Czito BG, Hurwitz HI, Clough RW, Tyler DS, Morse MA, Clary BM, et al. Adjuvant externalbeam radiotherapy with concurrent chemotherapy after resection of primary gallbladder carcinoma: a 23-year experience. Int J Radiat Oncol Biol Phys 2005; 62: 1030-4.

7. Wang SJ, Fuller CD, Kim JS, Sittig DF, Thomas CR Jr, Ravdin PM. Prediction model for estimating the survival benefit of adjuvant radiotherapy for gallbladder cancer. J Clin Oncol 2008; 26: 2112-7.

8. Gunderson LL, Martenson JA, Smalley SR, Garton GR. Upper gastrointestinal cancers: rationale, results, and techniques of treatment. Front Radiat Ther Oncol 1994; 28: 121-39.

9. Coia LR, Myerson RJ, Tepper JE. Late effects of radiation therapy on the gastrointestinal tract. Int J Radiat Oncol Biol Phys 1995; 31: 1213-36. 
10. von Bultzingslowen I, Brennan MT, Spijkervet FK, Logan R, Stringer A, Raber-Durlacher JE, et al. Growth factors and cytokines in the prevention and treatment of oral and gastrointestinal mucositis. Support Care Cancer 2006; 14: 519-27.

11. Fajardo LF. Morphology of radiation effects on normal tissues. In: Perez CA, Brady LW, editors. Principles and practice of radiation oncology. 3rd ed. Philadelphia: Lippincott-Raven; c1998. p. $143-54$.

12. Hauer-Jensen M, Wang J, Denham JW. Bowel injury: current and evolving management strategies. Semin Radiat Oncol 2003; 13: 357-71.

13. Letschert JG, Lebesque JV, Aleman BM, Bosset JF, Horiot JC, Bartelink $\mathrm{H}$, et al. The volume effect in radiation-related late small bowel complications: results of a clinical study of the EORTC Radiotherapy Cooperative Group in patients treated for rectal carcinoma. Radiother Oncol 1994; 32: 116-23.

14. Regine WF, Winter KA, Abrams RA, Safran H, Hoffman JP, Konski A, et al. Fluorouracil vs gemcitabine chemotherapy before and after fluorouracil-based chemoradiation following resection of pancreatic adenocarcinoma: a randomized controlled trial. JAMA 2008; 299(9):1019-26. Erratum in: JAMA 2008; 299(16): 1902.

15. Cahan WG, Woodard HQ, Higinbotham NL, Stewart FW, Coley BL. Sarcoma arising in irradiated bone: report of 11 cases. Cancer 1948; 1: 3-29.

16. Morgan MA, Moutos DM, Pippitt CH Jr, Suda RR, Smith JJ, Thurnau GR. Vaginal and bladder angiosarcoma after therapeutic irradiation. South Med J 1989; 82: 1434-6. 